\title{
AVALIAÇÃo DE UM MODELO DE DISTRIBUIÇÃO DIAMÉTRICA AJUSTADO PARA POVOAMENTOS DE Eucalyptus sp. SUBMETIDOS A DESBASTE ${ }^{1}$
}

\author{
Helio Garcia Leite ${ }^{2}$, Gilciano Saraiva Nogueira ${ }^{3}$, João Carlos Chagas Campos², Agostinho Lopes de \\ Souza $^{2}$ e Andrelino Carvalho ${ }^{4}$
}

\begin{abstract}
RESUMO - O principal objetivo deste estudo foi avaliar as estimativas geradas por um modelo de distribuição diamétrica, do ponto de vista biológico. Também foi objetivo avaliar a eficiência do método dos ingressos porcentuais para determinação da idade técnica do primeiro e do segundo desbaste em plantação de eucalipto. A análise qualitativa do modelo consistiu em avaliar os resultados obtidos pelo método dos ingressos porcentuais, o comportamento das distribuições diamétricas projetadas para idades futuras e o comportamento das distribuições diamétricas remanescentes, após algumas simulações de desbaste, do ponto de vista da teoria, da lógica e do realismo biológico. Pôde-se inferir que o modelo de distribuição diamétrica proposto é biologicamente correto e consistente. A estagnação do crescimento é mais rápida após o desbaste. Concluiu-se, ainda, que o método dos ingressos porcentuais é adequado para determinar a idade técnica do primeiro e do segundo desbaste. $\mathrm{O}$ modelo avaliado permite simular diferentes cenários, gerando estimativas consistentes, podendo ser aplicado sem restrições, ou seja, podem ser simulados cenários que contemplem, ou não, as condições observadas no campo.
\end{abstract}

Palavras-chave: Eucalipto, distribuição diamétrica, desbaste, método dos ingressos porcentuais e avaliação de modelo.

\section{EVALUATION OF AN ADJUSTED DIAMETRIC DISTRIBUTION MODEL FOR THINNED Eucalyptus sp. STANDS}

\begin{abstract}
The main objective of this study was to evaluate the estimates generated by a diametric distribution model, under the biologic point of view. Another objective was to evaluate the efficiency of the percentage of entries method to determine the technical age for the first and second thinning in eucalypt stands. The qualitative evaluation of the model was carried out through the evaluation of the results obtained by the percentage of entries method, the behavior of the diametric distributions projected for the future, and the behavior of the remaining distributions after some thinning simulations, theoretically, logically and biologically speaking. According to the results it was concluded that the diametric distribution model proposed is biologically correct and consistent. Growth stagnation is stimulated after thinning. It also was concluded that the percentage of entries method is suitable to determine the technical age for the first and second thinning. The model evaluated allows the simulation of various situations, generating consistent estimates and it can be used without restrictions, that is, situations can be simulated with or without the conditions observed in the field.
\end{abstract}

Key words: Eucalypt, diametric distribution model, thinning, percentage of entries method and model evaluation.

\footnotetext{
${ }^{1}$ Recebido em 01.06.2004 e aceito para publicação em 25.11.2004.

2 Departamento de Engenharia Florestal da Universidade Federal de Viçosa, 36570-000 Viçosa-MG.

${ }^{3}$ Departamento de Engenharia Florestal das Faculdades Federais Integradas de Diamantina (FAFEID).

${ }^{4}$ Bahia Pulp.
} 


\section{INTRODUÇÃO}

Um modelo de crescimento e produção deve representar a realidade do sistema ou fenômeno considerado. Sua avaliação compreende interpretações de ordem qualitativa, que trata de aspectos como o realismo biológico do modelo, e de ordem quantitativa, que trata de testes estatísticos e comparação de predições com observações independentes (CAMPOS e LEITE, 2002). Dentre os modelos clássicos de crescimento e produção, os de distribuição diamétrica são mais adequados para analisar desbaste, uma vez que possibilitam a avaliação econômica de multiprodutos, além de serem bem mais flexíveis (BURKHART et al., 1981). Em função desse argumento, foi proposto e ajustado um modelo de distribuição diamétrica utilizando dados de povoamentos desbastados de eucalipto. Neste trabalho, esse modelo é avaliado biologicamente, sendo testada a seguinte hipótese: $\mathrm{H}_{0}$ : as estimativas geradas pelo modelo proposto seguem uma tendência lógica esperada do ponto de vista biológico! O principal objetivo deste estudo foi avaliar essa hipótese. Também foi objetivo avaliar a eficiência do método dos ingressos porcentuais para determinação da idade técnica do primeiro e do segundo desbaste em plantação de eucalipto.

\section{MATERIAL E MÉTODOS}

\subsection{Distribuição diamétrica}

O modelo de distribuição diamétrica utilizado neste estudo é composto pelas equações seguintes:

$$
\begin{aligned}
& d \min _{2}=d \min _{1} \mathrm{e}^{\left(-(-0,006786)\left(I_{2}^{0,862014}-I_{1}^{0,862014}\right)\right),} R_{\hat{y}}=0,971 \\
& \operatorname{Ln} \gamma_{2}=\operatorname{Ln} \gamma_{1} \mathrm{e}^{\left(-(111,9299)\left(I_{2}^{0,001853}-I_{1}^{0,001853}\right)\right)}, R_{\hat{y}}=0,980 \\
& d \max _{2}=\operatorname{dmax}_{1}\left(\frac{I_{1}}{I_{2}}\right)+2,040634\left(1-\frac{I_{1}}{I_{2}}\right) \beta_{1}, R_{\hat{y}}=0,958 \\
& \beta_{2}=\beta_{1}\left(\frac{I_{1}}{I_{2}}\right)+1,042851\left(1-\frac{I_{1}}{I_{2}}\right) d \max _{2}, \quad R_{\hat{y}}=0,982
\end{aligned}
$$

Antes do desbaste:

$$
N_{2}=N_{1} \mathrm{e}^{\left(-(273,8150)\left(I_{2}^{0,000016}-I_{1}^{0,000016}\right)\right)}, R_{\hat{y}}=0,997
$$

Após o desbaste: $N_{2}=N_{1}$,

em que:

R. Árvore, Viçosa-MG, v.29, n.2, p.271-280, 2005
$I_{2}=$ idade futura, em meses; $I_{1}=$ idade atual, em meses; $\gamma_{2}=$ parâmetro de forma da função Weibull em uma idade futura; $\gamma_{1}=$ parâmetro de forma da função Weibull em uma idade atual; $\beta_{2}=$ parâmetro de escala da função Weibull em uma idade futura; $\beta_{1}=$ parâmetro de escala da função Weibull em uma idade atual; $\operatorname{dmax}_{2}=$ diâmetro máximo em uma idade futura, em cm; dmax $_{1}=$ diâmetro máximo em uma idade atual, em cm; $d \min _{2}=$ diâmetro mínimo em uma idade futura, em cm; $d \min _{1}=$ diâmetro mínimo em uma idade atual, em cm; $N_{2}=$ número de árvores por ha em uma idade futura; $N_{1}=$ número de árvores por ha em uma idade atual; e $L n=\log$ aritmo neperiano.

O limite superior da distribuição teórica dos diâmetros por classe foi determinado pela estimativa do diâmetro máximo, acrescentada de duas classes de diâmetro. Assim, se o diâmetro máximo estimado for de $19,3 \mathrm{~cm}$, o ponto de truncamento da distribuição à direita será na classe de $21,5 \mathrm{~cm}$. Essa decisão foi tomada com base em análises preliminares que indicaram estimativas precisas da produção total por unidade de área. O diâmetro máximo foi estimado pela equação do $\operatorname{dmax}_{2}$ (equação 3). Considerou-se como limite inferior da distribuição o diâmetro mínimo do povoamento. Para projetar esse limite para idades futuras, empregou-se a equação do $\operatorname{dmin}_{2}$ (equação 1 ).

Cabe observar que o sistema não considera mortalidade após o desbaste, o que satisfaz um dos objetivos deste tratamento silvicultural, que é a eliminação da mortalidade natural das árvores. Antes do desbaste foi utilizado um modelo proposto por Pienaar e Schiver (1981) para estimar a sobrevivência.

Também fazem parte do modelo de distribuição diamétrica as seguintes equações:

$$
\begin{aligned}
& \operatorname{LnHd}=3,523560-24,535140(I)^{-1}, \bar{r}^{2}=0,93 \\
& H t=-50,082731-0,053019(I)+0,757315(S)+8,514866 \operatorname{Ln}(I \cdot d a p), \\
& \overline{\mathrm{R}}^{2}=0,972 \\
& \hat{V}=0,000087(\text { dap })^{1,551523}(H t)^{1,125578} \mathrm{e}^{-2,466217\left(\frac{T x}{d a p}\right)}\left[1-\left(\frac{d}{d a p}\right)^{1+0,224175(d)}\right], \\
& R_{\hat{y}}=0,99
\end{aligned}
$$

em que:

$H d=$ altura dominante, em $\mathrm{m} ; I=$ idade, em meses; $H t=$ altura total da árvore, em $\mathrm{m} ;$ dap $=$ diâmetro a $1,30 \mathrm{~m}$ de altura, em cm; $S=$ índice de local; $\hat{V}=$ volume 
comercial com e sem casca por árvore, em $\mathrm{m}^{3} ; T x=$ variável binária, sendo $T x=0$ para volume com casca e $T x=1$ para volume sem casca; e $d=$ diâmetro mínimo comercial.

Os dados utilizados para ajustar o modelo pertencem a um experimento sobre desbastes, instalado em povoamentos do híbrido Eucalyptus grandis $\mathrm{x}$ Eucalyptus urophylla, na empresa Copener Florestal, na região nordeste do Estado da Bahia. As medições foram realizadas aos $27,40,50,58,61,76,87,101 \mathrm{e}$ 112 meses, em 48 parcelas permanentes retangulares, com área útil de aproximadamente $2.600 \mathrm{~m}^{2}$. O primeiro desbaste foi feito aos 58 meses, considerando-se diferentes porcentagens de redução da área basal (redução de 20, 35 e 50\% na área basal presente, removendo os piores indivíduos).

\subsection{Projeção da distribuição diamétrica e idade técnica de desbaste}

A projeção da distribuição diamétrica foi realizada a partir de uma distribuição em uma idade atual $\left(I_{1}\right)$ para uma idade futura $\left(I_{2}\right)$, partindo-se de uma distribuição inicial observada. O povoamento florestal antes do desbaste é distinto do povoamento após o desbaste, ou seja, após o desbaste o povoamento apresenta novas tendências de crescimento e produção. Portanto, considerou-se uma distribuição diamétrica inicial observada antes do primeiro desbaste e outra após o desbaste. Antes do desbaste, utilizaram-se as distribuições observadas nas idades de 27,40 e 50 meses; de posse dos dados da última medição (50 meses), projetaram-se as distribuições para idades futuras. Após o desbaste, utilizaram-se as distribuições observadas nas idades de 61, 76, 87, 101 e 112 meses, e projetaramse as distribuições para idades futuras, a partir dos dados da última medição (112 meses).

O momento ótimo de desbastar foi determinado empregando-se o Método dos Ingressos Porcentuais (MIP), proposto por Garcia (1999) e utilizado por Nogueira et al. (2001). Esse método consiste na análise da dinâmica de crescimento e na avaliação do ingresso das árvores em sucessivas classes de diâmetro.

O ingresso porcentual (IP) das árvores em sucessivas classes de diâmetros foi calculado através da diferença entre distribuições de diâmetros em duas ocasiões diferentes $\left(F\left(X_{2}\right)-F\left(X_{1}\right)\right.$ ), utilizando um aplicativo específico, codificado em linguagem Pascal, desenvolvido por Garcia (1999). O aplicativo fornece os ingressos porcentuais, utilizando como inputs o número de árvores e as estimativas dos parâmetros da função Weibull, nas idades sucessivas. Os valores de $\mathrm{F}\left(\mathrm{X}_{\mathrm{i}}\right)$ foram obtidos empregando a forma acumulativa da função Weibull: $F\left(x_{\mathrm{i}}\right)=1-\mathrm{e}^{-\left(\frac{x}{\beta}\right)^{\gamma}}$.

De acordo com o MIP, a idade técnica do desbaste ocorre quando o ingresso de árvores em sucessivas classes de diâmetros não for mais significativo. Para determinar essa idade foi empregado o modelo expolinear (GOUDRIAAN, 1994), cuja relação funcional é: $I P M=\frac{\theta_{1}}{\theta_{2}} \operatorname{Ln}\left[1+\mathrm{e}^{\theta_{2}(I-I T D)}\right]+\varepsilon$ em que:

$I P M=$ ingresso porcentual médio mensal de árvores; $I P=$ ingresso porcentual de árvores; $I=$ idade do povoamento, em meses; $\theta_{1}=$ parâmetro que indica o crescimento máximo absoluto do ingresso porcentual de árvores na fase linear da curva; $\theta_{2}=$ parâmetro que indica o crescimento máximo relativo do ingresso porcentual de árvores na fase exponencial da curva; $I T D=$ parâmetro que corresponde à idade técnica de desbaste; e $\varepsilon=$ erro aleatório, $\varepsilon \sim \operatorname{NID}\left(0, \sigma^{2}\right)$.

Os ingressos porcentuais foram divididos pela idade, obtendo-se o incremento porcentual médio mensal. O IPM foi empregado para que as fases linear e exponencial fossem bem caracterizadas e, assim, facilitassem o ajuste do modelo expolinear. O modelo foi ajustado através de regressão não-linear, utilizando o procedimento iterativo Quasi-Newton.

A idade técnica do primeiro e do segundo desbaste foi determinada para diferentes classes de produtividade. No segundo desbaste foram consideradas também várias porcentagens de redução de área basal.

\subsection{Simulação de desbastes}

Para avaliar a flexibilidade do modelo foram simulados desbastes em diferentes idades, com distintas porcentagens de redução da área basal, em locais com capacidades produtivas diferentes e para diversos usos da madeira. A simulação foi realizada considerando a distribuição média dos diâmetros na idade técnica do primeiro desbaste. Para isso, projetou-se a distribuição dos diâmetros da última medição antes do desbaste para a idade recomendada, por meio do MIP.

R. Árvore, Viçosa-MG, v.29, n.2, p.271-280, 2005 
Foi simulada a colheita de árvores, com base na redução da área basal, nas classes de diâmetros inferiores (desbaste seletivo). As áreas basais das árvores colhidas foram computadas e somadas até atingir a porcentagem de diminuição de área basal desejada. Dessa forma, em determinada classe apenas parte do número de árvores poderá ser colhida, já que a porcentagem de redução desejada poderá ser atingida.

\section{RESULTADOS E DISCUSSÃO}

\subsection{Projeção da distribuição diamétrica}

Um exemplo de projeção das estimativas de $d m a x$,

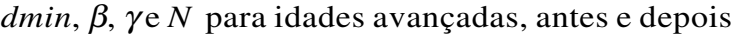
do primeiro desbaste, é apresentado no Quadro 1. Antes do primeiro desbaste, considerou-se a idade 27 como $I_{1}$. Assim, $\beta_{1}$ foi igual a $9,089, \gamma_{1}$ igual a 6,523, dmax $_{1}$ igual a $13, d \min _{1}$ igual a 2,5 e $N_{1}$ igual a 1307 . De posse desses valores observados (valores médios), projetaramse essas variáveis para $I_{2}$ (39 meses). Com as estimativas das variáveis em $I_{2}$, foram projetadas as estimativas das variáveis para $I_{3}$ (51 meses). Assim, de 12 em 12 meses, projetou-se até a idade de 135 meses. Após o primeiro desbaste, procedeu-se da mesma forma, considerando a idade de 61 meses como $I_{1}$, e as distribuições foram projetadas até a idade de 169 meses.

Com as estimativas do número de árvores e dos parâmetros da função Weibull, estimou-se a freqüência por hectare em cada classe de diâmetro, para todas as idades consideradas. Na Figura 1 são apresentadas as distribuições diamétricas projetadas, considerandose os dados iniciais observados antes e depois do primeiro desbaste.

Ao longo do tempo, as distribuições diamétricas se achatam e deslocam para a direita, sendo esse o comportamento natural da distribuição diamétrica em povoamentos eqüiâneos. Com isso, pode-se inferir que a redistribuição do número de árvores por classe de diâmetro ao longo do tempo, obtida pelo modelo de distribuição diamétrica, está de acordo com a teoria. Outro aspecto que também está conforme a teoria é o comportamento do ingresso de árvores em sucessivas classes de diâmetros. Observou-se que as áreas correspondentes aos ingressos diminuem com o passar do tempo, antes e depois do desbaste. Foram verificadas também tendências de crescimento diferentes antes e depois do desbaste (Figura 1).

\subsection{Idade técnica de desbaste}

As áreas correspondentes aos ingressos de árvores em novas classes de diâmetros (Figura 1) diminuem significativamente ao longo do tempo. A partir de determinado momento, esse ingresso não é mais significativo, indicando que o espaço disponível não é mais suficiente para o crescimento significativo das árvores.

Para calcular os ingressos porcentuais, foi necessário projetar as distribuições diamétricas de todas as parcelas, utilizando-se como distribuição diamétrica inicial a última medição. Assim, antes do desbaste, empregaram-se as distribuições observadas nas idades de 27, 40 e 50 meses; de posse dos dados da última medição (50 meses), projetou-se a distribuição para idades futuras. Após o desbaste, usaram-se as distribuições observadas nas idades de 61, 76, 87, 101 e 112 meses, e projetouse a distribuição para idades futuras, a partir dos dados da última medição (112 meses). Os ingressos porcentuais foram divididos pela idade, obtendo-se o incremento

Quadro 1 - Estimativas obtidas com o modelo de distribuição diamétrica

Table 1 - Estimates obtained from the diametric distribution model

\begin{tabular}{lccccc}
\hline $\begin{array}{l}\text { Idade } \\
(\text { meses })\end{array}$ & $\begin{array}{c}\text { dmax } \\
(\mathrm{cm})\end{array}$ & $\begin{array}{c}\text { dmin } \\
(\mathrm{cm})\end{array}$ & $\beta$ & $\gamma$ & $N$ \\
\hline \multicolumn{5}{c}{ Antes do $1^{\circ}$ desbaste } \\
\hline $27^{*}$ & 13,0 & 2,5 & 9,089 & 6,523 & 1307 \\
39 & 14,7 & 2,6 & 11,011 & 5,679 & 1305 \\
51 & 16,5 & 2,7 & 12,477 & 5,166 & 1303 \\
63 & 18,2 & 2,8 & 13,723 & 4,812 & 1302 \\
75 & 19,8 & 2,9 & 14,830 & 4,549 & 1301 \\
87 & 21,2 & 3,1 & 15,840 & 4,343 & 1300 \\
99 & 22,6 & 3,2 & 16,775 & 4,177 & 1299 \\
111 & 23,8 & 3,3 & 17,649 & 4,038 & 1299 \\
123 & 25,0 & 3,4 & 18,474 & 3,920 & 1298 \\
135 & 26,2 & 3,5 & 19,257 & 3,818 & 1298 \\
\hline \multicolumn{5}{c}{ Após o $1^{\circ}$ desbaste } & \\
\hline $61 *$ & 19,0 & 6,5 & 14,404 & 10,549 & 699 \\
73 & 20,7 & 6,8 & 15,586 & 9,672 & 699 \\
85 & 22,3 & 7,0 & 16,665 & 9,009 & 699 \\
97 & 23,7 & 7,3 & 17,665 & 8,485 & 699 \\
109 & 25,1 & 7,6 & 18,600 & 8,059 & 699 \\
121 & 26,4 & 7,9 & 19,481 & 7,703 & 699 \\
133 & 27,6 & 8,1 & 20,317 & 7,401 & 699 \\
145 & 28,7 & 8,4 & 21,115 & 7,141 & 699 \\
157 & 29,8 & 8,7 & 21,877 & 6,913 & 699 \\
169 & 30,9 & 9,0 & 22,610 & 6,711 & 699 \\
\hline
\end{tabular}

* Valores observados. 
porcentual médio mensal (IPM). As estimativas de IPM, antes e depois do desbaste, são apresentadas na Figura 2. É possível identificar visualmente o intervalo que contempla a idade técnica de desbaste (ITD). Antes do desbaste, essa idade estava entre 70 e 90 meses e após, entre 110 e 130 meses. A identificação exata da ITD foi feita através do ajuste do modelo expolinear.

O modelo expolinear foi ajustado para classes de produtividade e porcentagem de desbastes diferentes. As equações ajustadas com os respectivos coeficientes de correlação $\left(R_{\hat{y}}\right)$ antes e depois do primeiro desbaste, considerando-se classes de produtividades extremas (classes I e III) e a redução de 20, 35 e 50\% na área basal, são apresentadas no Quadro 2.
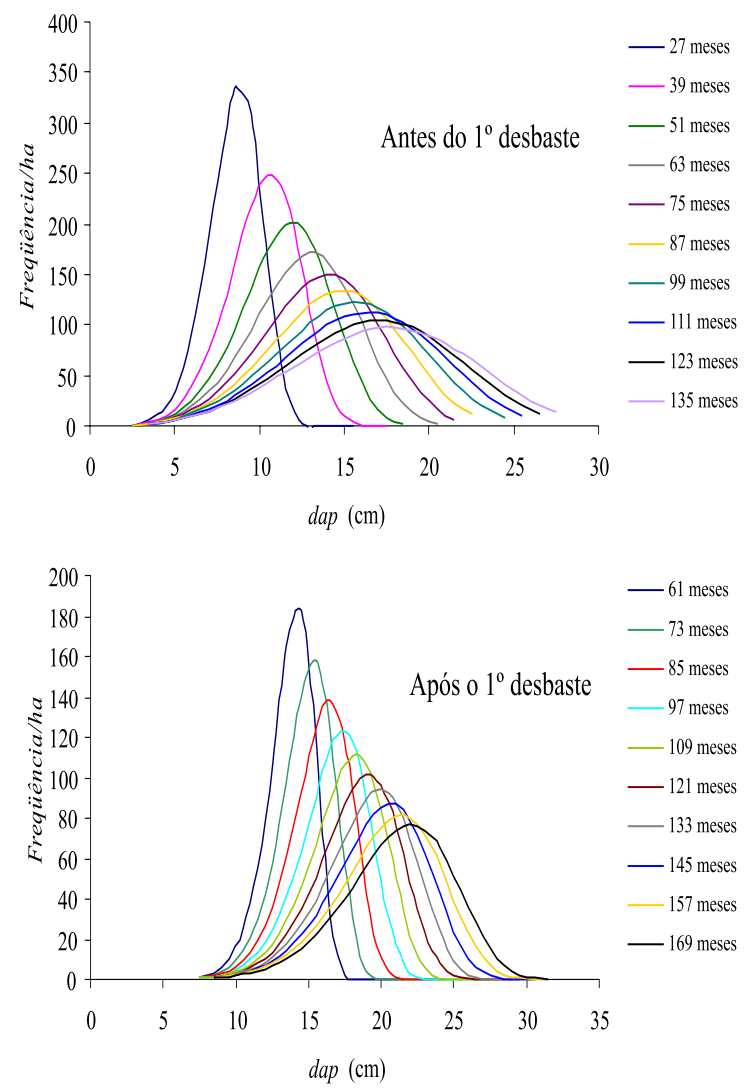

Figura 1 - Distribuições diamétricas estimadas antes e depois do primeiro desbaste.

Figure 1 - Diametric distributions estimated before and after thinning.

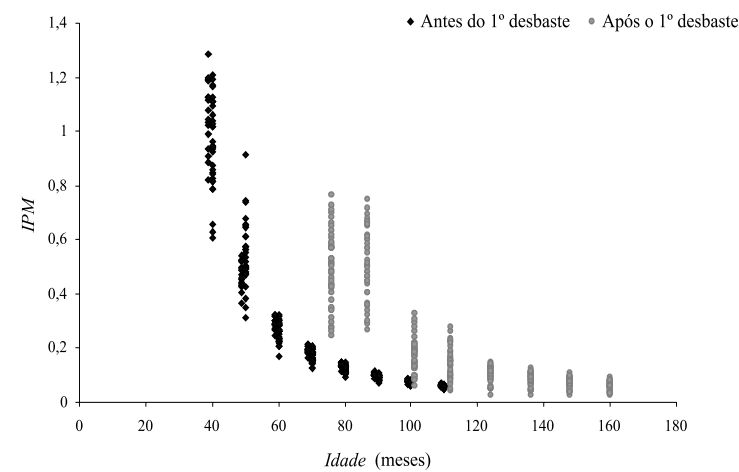

Figura 2 - Ingresso porcentual médio $(I P M)$ antes e depois do primeiro desbaste.

Figure 2-Mean percentage of entries (IPM) before and after thinning.

As ITDs obtidas estão dentro do intervalo identificado visualmente, mostrando que o modelo expolinear é adequado para determinar a idade técnica do primeiro e do segundo desbaste. Outra constatação é que o sistema de equações proposto para redistribuir as distribuições diamétricas é lógico do ponto de vista biológico, uma vez que as seguintes tendências ou pressuposições foram satisfeitas:

- A classe de produtividade maior resultou em uma idade técnica de desbaste menor.

- Quanto maior a capacidade produtiva de um local, maior a taxa de crescimento das árvores.

- Quanto maior a taxa de crescimento das árvores, mais cedo o povoamento inicia o processo de competição entre as árvores, o que resulta na estagnação do crescimento.

- A idade técnica do primeiro desbaste para a classe de produtividade III foi de 88 meses e para a classe de produtividade I, 75 meses. Após o desbaste, essa tendência também foi verificada, tanto considerando todas as porcentagens de redução de área basal (classe III, ITD $=119$ meses; e classe I, ITD $=110$ meses) quanto cada porcentagem separadamente.

- Quanto maior a porcentagem de redução de área basal, maior a idade técnica de desbaste. Em outras palavras, quanto menor o número de árvores remanescentes, maior o espaço para o crescimento e, conseqüentemente, menor a competição entre as árvores e maior a ITD. Na classe de produtividade III, as idades

R. Árvore, Viçosa-MG, v.29, n.2, p.271-280, 2005 
técnicas do segundo desbaste foram de 112, 118 e 124 meses, considerando-se, respectivamente, a redução de 20,35 e $50 \%$ da área basal. Já na classe de produtividade I, as ITDs foram de 104, 110 e 118 meses, levando-se em conta as mesmas reduções de área basal.

- Após o desbaste ocorre aumento na taxa de crescimento das árvores remanescentes. Com o desbaste, os fatores de produção ficam à disposição das árvores dominantes e co-dominantes (árvores remanescentes), que são mais eficientes na relação fotossínteses/ respiração, aumentando a velocidade de crescimento dessas árvores. Essa tendência verificada pode ser observada na Figura 2, que mostra a relação entre os ingressos porcentuais antes e depois do desbaste com a idade. Observou-se que após o desbaste houve aumento significativo nos ingressos porcentuais, ou seja, ocorreu a retomada do crescimento das árvores remanescentes.
- A estagnação do crescimento é mais rápida após o desbaste. Conforme mencionado, a estagnação é diretamente proporcional à taxa de crescimento; como essa taxa é maior após o desbaste, conseqüentemente a estagnação acontece mais cedo. Para exemplificar, na classe I a diferença entre a idade técnica do segundo desbaste e a do primeiro foi de 52 meses ( 110 - 58 meses), ou seja, antes do desbaste foram necessários 75 meses para iniciar o processo de estagnação do crescimento, enquanto após se necessitou de apenas 52 meses.

\subsection{Simulação de desbastes}

Para verificar a flexibilidade do modelo na simulação de desbaste, foram consideradas as idades técnicas recomendadas para as diferentes capacidades produtivas. Assim, a partir da distribuição média dos diâmetros nas classes de produtividade extremas (classes I e III), aos 50 meses, projetaram-se as variáveis do modelo de distribuição diamétrica nas idades de 75 e 88 meses, nas classes I e III, respectivamente (Quadro 3).

Quadro 2 - Equações expolineares e idades técnicas de desbaste de diferentes classes de produtividade e porcentagens de desbaste

Table 2 - Expolinear equations and technical ages for the thinning of different site quality classes and percentages of thinning

\begin{tabular}{|c|c|c|c|c|}
\hline $\begin{array}{l}\text { Classe } \\
\text { de Produção }\end{array}$ & $\begin{array}{c}\% \text { de } \\
\text { Redução } \\
\text { de } B / h a\end{array}$ & Equação Expolinear & $R_{\hat{y} y}$ & $\begin{array}{c}I T D \\
\text { (meses) }\end{array}$ \\
\hline & & Antes do $1^{\circ}$ desbaste & & \\
\hline III & - & $I P M=\frac{-35,0266}{-0,0520} \operatorname{Ln}[1+\exp (-0,0520($ Idade $-(-87,9010)))]$ & 0,966 & 88 \\
\hline \multirow[t]{2}{*}{ I } & - & $I P M=\frac{-66,3441}{-0,0604} \operatorname{Ln}[1+\exp (-0,0604($ Idade $-(-75,0600)))]$ & 0,975 & 75 \\
\hline & & \multicolumn{3}{|l|}{ Após o $1^{\circ}$ desbaste } \\
\hline \multirow[t]{4}{*}{ III } & - & $I P M=\frac{-0,0117}{-5,1027} \operatorname{Ln}[1+\exp (-5,1027($ Idade $-(119,2083)))]$ & 0,822 & 119 \\
\hline & 20 & $I P M=\frac{-0,0112}{-0,0556} \operatorname{Ln}[1+\exp (-0,0556(\operatorname{Idade}-(111,7903)))]$ & 0,864 & 112 \\
\hline & 35 & $I P M=\frac{-0,0144}{-4,0592} \operatorname{Ln}[1+\exp (-4,0592($ Idade $-(118,5227)))]$ & 0,878 & 118 \\
\hline & 50 & $I P M=\frac{-295,615}{-0,0457} \operatorname{Ln}[1+\exp (-0,0457($ Idade $-(-124,157)))]$ & 0,961 & 124 \\
\hline \multirow[t]{4}{*}{ I } & - & $I P M=\frac{-5,2226}{-0,0319} \operatorname{Ln}[1+\exp (-0,0319($ Idade $-(-110,255)))]$ & 0,917 & 110 \\
\hline & 20 & $I P M=\frac{-5,3505}{-0,0332} \operatorname{Ln}[1+\exp (-0,0332($ Idade $-(-104,301)))]$ & 0,892 & 104 \\
\hline & 35 & $I P M=\frac{-5,5493}{-0,0313} \operatorname{Ln}[1+\exp (-0,0313($ Idade $-(-110,637)))]$ & 0,979 & 111 \\
\hline & 50 & $I P M=\frac{-7,3784}{-0,0319} \operatorname{Ln}[1+\exp (-0,0319($ Idade $-(-118,508)))]$ & 0,949 & 118 \\
\hline
\end{tabular}

R. Árvore, Viçosa-MG, v.29, n.2, p.271-280, 2005 
Quadro3 - Estimativas obtidas nas idades técnicas de desbaste, a partir da distribuição observada aos 50 meses, considerando-se as classes de produtividade I e III

Table 3 - Production estimates obtained in the technical ages for thinning, based on the distribution observed at 50 months, considering the site classes I and III

\begin{tabular}{|c|c|c|c|c|c|}
\hline $\begin{array}{l}\text { Idade } \\
\text { (meses) }\end{array}$ & $\begin{array}{c}d \operatorname{dmax} \\
(\mathrm{cm})\end{array}$ & $\beta$ & $\gamma$ & $N$ & $\begin{array}{l}d m i n \\
(\mathrm{~cm})\end{array}$ \\
\hline & \multicolumn{5}{|c|}{ Classe I - Site 25} \\
\hline $50 *$ & 19,0 & 12,729 & 4,941 & 1237 & 2,5 \\
\hline \multirow[t]{2}{*}{75} & 22,0 & 15,797 & 4,340 & 1235 & 2,6 \\
\hline & \multicolumn{5}{|c|}{ Classe III - Site 22} \\
\hline $50 *$ & 18,0 & 11,830 & 5,776 & 1309 & 3,5 \\
\hline 88 & 22,2 & 15,985 & 4,751 & 1306 & 3,7 \\
\hline
\end{tabular}

* Valores observados.

Com base na redução da área basal desejada, eliminou-se parte do número de árvores na distribuição diamétrica. Foram colhidas as árvores das menores classes de diâmetros até atingir a redução desejada. Esse tipo de desbaste é denominado seletivo. Assim, utilizando o sistema de equações proposto, a equação de altura total e a equação de múltiplos volumes foram construídos os Quadros 4 e 5. Considerou-se a produção volumétrica para energia (diâmetro mínimo comercial de $4 \mathrm{~cm}$ ) e para serraria (diâmetro mínimo comercial de $15 \mathrm{~cm}$ ), os índices de local extremos e um desbaste de $40 \%$ da área basal.
Conforme mencionado, as áreas basais das árvores colhidas são computadas e somadas até atingir a porcentagem de redução da área basal desejada. Assim, em determinada classe, parte do número total de árvores poderá ser colhida apenas para completar a redução desejada. Por exemplo, nos Quadros 4 e 5, para a classe de produtividade I a área basal desejada foi atingida na classe 15,5 . Nessa classe, foram mantidas 73 e colhidas 53 árvores.

De acordo com as estimativas apresentadas nos Quadros 4 e 5, pode-se inferir que o sistema de equações do modelo de distribuição diamétrica é adequado para predizer a produção volumétrica (total, remanescente e desbastada). O modelo foi lógico e biologicamente correto. Isso pode ser verificado através de alguns aspectos - por exemplo, o índice de local maior proporcionou maior produtividade volumétrica. Podese observar que, apesar de o povoamento na classe de produtividade I apresentar número menor de árvores e ser desbastado mais cedo do que o povoamento na classe III, as produtividades obtidas foram praticamente as mesmas. Outro exemplo está relacionado com o volume comercial: muitas árvores desbastadas são aproveitadas para energia, enquanto poucas o são para serraria, o que está correto, já que no primeiro desbaste o povoamento apresenta poucas árvores com dimensões apropriadas para serraria.

Quadro 4 - Estimativas de produção comercial obtidas com um desbaste seletivo de $40 \%$ da área basal aos 88 e 75 meses, nas classes de local III e I

Table 4 -Commercial production estimates obtained from a selective thinning of $40 \%$ of the basal area at 88 and 75 months, in the site classes I and III

\begin{tabular}{|c|c|c|c|c|c|c|c|c|c|c|c|c|c|c|}
\hline $\begin{array}{l}\text { Idade } \\
\text { (meses) }\end{array}$ & $\begin{array}{l}\text { dap } \\
(\mathrm{cm})\end{array}$ & $\begin{array}{c}\mathrm{N} \\
\text { total } \\
\text { (Aro/ha) }\end{array}$ & $\begin{array}{c}\mathrm{B} \\
\text { total } \\
\left(\mathrm{m}^{2} / \mathrm{ha}\right)\end{array}$ & $\begin{array}{c}\mathrm{Ht} \\
\text { total } \\
(\mathrm{m})\end{array}$ & $\begin{array}{c}\mathrm{V} 4 \mathrm{cc} \\
\text { total } \\
\left(\mathrm{m}^{3} / \mathrm{ha}\right)\end{array}$ & $\begin{array}{c}\mathrm{V} 4 \mathrm{sc} \\
\text { total } \\
\left(\mathrm{m}^{3} / \mathrm{ha}\right)\end{array}$ & $\begin{array}{c}\mathrm{N} \\
\text { reman. } \\
\text { (Aro/ha) }\end{array}$ & $\begin{array}{c}\mathrm{B} \\
\text { reman. } \\
\left(\mathrm{m}^{2} / \mathrm{ha}\right)\end{array}$ & $\begin{array}{c}\mathrm{V} 4 \mathrm{cc} \\
\text { reman. } \\
\left(\mathrm{m}^{3} / \mathrm{ha}\right)\end{array}$ & $\begin{array}{c}\mathrm{V} 4 \mathrm{sc} \\
\text { reman. } \\
\left(\mathrm{m}^{3} / \mathrm{ha}\right)\end{array}$ & $\begin{array}{c}\mathrm{N} \\
\text { desbas. } \\
\text { (Aro/ha) }\end{array}$ & $\begin{array}{c}\text { B } \\
\text { desbas. } \\
\left(\mathrm{m}^{3} / \mathrm{ha}\right)\end{array}$ & $\begin{array}{c}\text { V4cc } \\
\text { desbas. } \\
\left(\mathrm{m}^{3} / \mathrm{ha}\right)\end{array}$ & $\begin{array}{r}\text { V4sc } \\
\text { desbas. } \\
\left(\mathrm{m}^{3} / \mathrm{ha}\right)\end{array}$ \\
\hline & & \multicolumn{13}{|c|}{ Classe III - Site 22} \\
\hline 88 & 3,5 & 1 & 0,001 & 10,7 & 0,00 & 0,00 & & & & & 1 & 0,001 & 0,00 & 0,00 \\
\hline 88 & 4,5 & 3 & 0,005 & 12,8 & 0,01 & 0,01 & & & & & 3 & 0,005 & 0,01 & 0,01 \\
\hline 88 & 5,5 & 7 & 0,017 & 14,6 & 0,08 & 0,05 & & & & & 7 & 0,017 & 0,08 & 0,05 \\
\hline 88 & 6,5 & 13 & 0,044 & 16,0 & 0,29 & 0,20 & & & & & 13 & 0,044 & 0,29 & 0,20 \\
\hline 88 & 7,5 & 22 & 0,098 & 17,2 & 0,76 & 0,55 & & & & & 22 & 0,098 & 0,76 & 0,55 \\
\hline 88 & 8,5 & 35 & 0,197 & 18,3 & 1,68 & 1,26 & & & & & 35 & 0,197 & 1,68 & 1,26 \\
\hline 88 & 9,5 & 51 & 0,360 & 19,2 & 3,28 & 2,53 & & & & & 51 & 0,360 & 3,28 & 2,53 \\
\hline 88 & 10,5 & 70 & 0,607 & 20,1 & 5,78 & 4,57 & & & & & 70 & 0,607 & 5,78 & 4,57 \\
\hline 88 & 11,5 & 92 & 0,951 & 20,8 & 9,35 & 7,54 & & & & & 92 & 0,951 & 9,35 & 7,54 \\
\hline 88 & 12,5 & 113 & 1,386 & 21,5 & 13,94 & 11,44 & & & & & 113 & 1,386 & 13,94 & 11,44 \\
\hline 88 & 13,5 & 131 & 1,879 & 22,2 & 19,22 & 16,01 & & & & & 131 & 1,879 & 19,22 & 16,01 \\
\hline Idade & dap & $\mathrm{N}$ & B & $\mathrm{Ht}$ & $\mathrm{V} 4 \mathrm{cc}$ & $\mathrm{V} 4 \mathrm{sc}$ & $\mathrm{N}$ & B & $\mathrm{V} 4 \mathrm{cc}$ & $\mathrm{V} 4 \mathrm{sc}$ & $\mathrm{N}$ & B & $\mathrm{V} 4 \mathrm{cc}$ & $\mathrm{V} 4 \mathrm{sc}$ \\
\hline
\end{tabular}


Quadro 4-Cont.

Table 4 - Cont.

\begin{tabular}{|c|c|c|c|c|c|c|c|c|c|c|c|c|c|c|}
\hline $\begin{array}{l}\text { Idade } \\
\text { (meses) }\end{array}$ & $\begin{array}{l}\text { dap } \\
(\mathrm{cm})\end{array}$ & $\begin{array}{c}\mathrm{N} \\
\text { total } \\
\text { (Aro/ha) }\end{array}$ & $\begin{array}{c}\mathrm{B} \\
\text { total } \\
\left(\mathrm{m}^{2} / \mathrm{ha}\right)\end{array}$ & $\begin{array}{l}\mathrm{Ht} \\
\text { total } \\
(\mathrm{m})\end{array}$ & $\begin{array}{l}\mathrm{V} 4 \mathrm{cc} \\
\text { total } \\
\left(\mathrm{m}^{3} / \mathrm{ha}\right)\end{array}$ & $\begin{array}{l}\text { V4sc } \\
\text { total } \\
\left(\mathrm{m}^{3} / \mathrm{ha}\right)\end{array}$ & $\begin{array}{c}\mathrm{N} \\
\text { reman. } \\
\text { (Aro/ha) }\end{array}$ & $\begin{array}{c}\text { B } \\
\text { reman. } \\
\left(\mathrm{m}^{2} / \mathrm{ha}\right)\end{array}$ & $\begin{array}{l}\mathrm{V} 4 \mathrm{cc} \\
\mathrm{reman} . \\
\left(\mathrm{m}^{3} / \mathrm{ha}\right)\end{array}$ & $\begin{array}{l}\mathrm{V} 4 \mathrm{sc} \\
\mathrm{reman} . \\
\left(\mathrm{m}^{3} / \mathrm{ha}\right)\end{array}$ & $\begin{array}{c}\mathrm{N} \\
\text { desbas. } \\
\text { (Aro/ha) }\end{array}$ & $\begin{array}{c}\text { B } \\
\text { desbas. } \\
\left(\mathrm{m}^{3} / \mathrm{ha}\right)\end{array}$ & $\begin{array}{l}\text { V4cc } \\
\text { desbas. } \\
\left(\mathrm{m}^{3} / \mathrm{ha}\right)\end{array}$ & $\begin{array}{r}\text { V4sc } \\
\text { desbas. } \\
\left(\mathrm{m}^{3} / \mathrm{ha}\right)\end{array}$ \\
\hline & & \multicolumn{13}{|c|}{ Classe III - Site 22} \\
\hline 88 & 14,5 & 143 & 2,362 & 22,8 & 24,46 & 20,63 & & & & & 143 & 2,362 & 24,46 & 20,63 \\
\hline 88 & 15,5 & 145 & 2,740 & 23,4 & 28,63 & 24,42 & 72 & 1,354 & 14,15 & 12,07 & 73 & 1,386 & 14,48 & 12,35 \\
\hline 88 & 16,5 & 136 & 2,912 & 23,9 & 30,63 & 26,38 & 136 & 2,912 & 30,63 & 26,38 & & & & \\
\hline 88 & 17,5 & 117 & 2,810 & 24,4 & 29,69 & 25,79 & 117 & 2,810 & 29,69 & 25,79 & & & & \\
\hline 88 & 18,5 & 91 & 2,435 & 24,9 & 25,81 & 22,59 & 91 & 2,435 & 25,81 & 22,59 & & & & \\
\hline 88 & 19,5 & 63 & 1,872 & 25,3 & 19,89 & 17,52 & 63 & 1,872 & 19,89 & 17,52 & & & & \\
\hline 88 & 20,5 & 38 & 1,259 & 25,8 & 13,39 & 11,87 & 38 & 1,259 & 13,39 & 11,87 & & & & \\
\hline 88 & 21,5 & 20 & 0,729 & 26,2 & 7,76 & 6,92 & 20 & 0,729 & 7,76 & 6,92 & & & & \\
\hline 88 & 22,5 & 9 & 0,357 & 26,5 & 3,80 & 3,41 & 9 & 0,357 & 3,80 & 3,41 & & & & \\
\hline 88 & 23,5 & 3 & 0,145 & 26,9 & 1,55 & 1,39 & 3 & 0,145 & 1,55 & 1,39 & & & & \\
\hline 88 & 24,5 & 1 & 0,048 & 27,3 & 0,51 & 0,46 & 1 & 0,048 & 0,51 & 0,46 & & & & \\
\hline \multirow[t]{2}{*}{ Total } & & 1305 & 23,2 & & 240,50 & 205,54 & 550 & 13,9 & 147,18 & 128,40 & 756 & 9,3 & 93,32 & 77,14 \\
\hline & \multicolumn{14}{|c|}{ Classe I - Site 25} \\
\hline 75 & 2,5 & 1 & 0,000 & 0,0 & 0,00 & 0,00 & & & & & 1 & 0,000 & 0,00 & 0,00 \\
\hline 75 & 3,5 & 2 & 0,002 & 12,3 & 0,00 & 0,00 & & & & & 2 & 0,002 & 0,00 & 0,00 \\
\hline 75 & 4,5 & 5 & 0,008 & 14,4 & 0,02 & 0,01 & & & & & 5 & 0,008 & 0,02 & 0,01 \\
\hline 75 & 5,5 & 10 & 0,024 & 16,2 & 0,13 & 0,08 & & & & & 10 & 0,024 & 0,13 & 0,08 \\
\hline 75 & 6,5 & 17 & 0,057 & 17,6 & 0,42 & 0,29 & & & & & 17 & 0,057 & 0,42 & 0,29 \\
\hline 75 & 7,5 & 27 & 0,120 & 18,8 & 1,03 & 0,74 & & & & & 27 & 0,120 & 1,03 & 0,74 \\
\hline 75 & 8,5 & 40 & 0,228 & 19,9 & 2,14 & 1,60 & & & & & 40 & 0,228 & 2,14 & 1,60 \\
\hline 75 & 9,5 & 56 & 0,395 & 20,8 & 3,94 & 3,04 & & & & & 56 & 0,395 & 3,94 & 3,04 \\
\hline 75 & 10,5 & 73 & 0,634 & 21,7 & 6,58 & 5,20 & & & & & 73 & 0,634 & 6,58 & 5,20 \\
\hline 75 & 11,5 & 91 & 0,948 & 22,4 & 10,13 & 8,18 & & & & & 91 & 0,948 & 10,13 & 8,18 \\
\hline 75 & 12,5 & 108 & 1,325 & 23,1 & 14,44 & 11,86 & & & & & 108 & 1,325 & 14,44 & 11,86 \\
\hline 75 & 13,5 & 121 & 1,730 & 23,8 & 19,13 & 15,94 & & & & & 121 & 1,730 & 19,13 & 15,94 \\
\hline 75 & 14,5 & 128 & 2,107 & 24,4 & 23,55 & 19,86 & & & & & 128 & 2,107 & 23,55 & 19,86 \\
\hline 75 & 15,5 & 126 & 2,386 & 25,0 & 26,86 & 22,91 & 73 & 1,384 & 15,58 & 13,29 & 53 & 1,002 & 11,29 & 9,63 \\
\hline 75 & 16,5 & 117 & 2,501 & 25,5 & 28,29 & 24,37 & 117 & 2,501 & 28,29 & 24,37 & & & & \\
\hline 75 & 17,5 & 100 & 2,411 & 26,0 & 27,37 & 23,77 & 100 & 2,411 & 27,37 & 23,77 & & & & \\
\hline 75 & 18,5 & 79 & 2,124 & 26,5 & 24,15 & 21,13 & 79 & 2,124 & 24,15 & 21,13 & & & & \\
\hline 75 & 19,5 & 57 & 1,694 & 26,9 & 19,28 & 16,99 & 57 & 1,694 & 19,28 & 16,99 & & & & \\
\hline 75 & 20,5 & 37 & 1,213 & 27,4 & 13,80 & 12,24 & 37 & 1,213 & 13,80 & 12,24 & & & & \\
\hline 75 & 21,5 & 21 & 0,772 & 27,8 & 8,78 & 7,83 & 21 & 0,772 & 8,78 & 7,83 & & & & \\
\hline 75 & 22,5 & 11 & 0,432 & 28,1 & 4,90 & 4,39 & 11 & 0,432 & 4,90 & 4,39 & & & & \\
\hline 75 & 23,5 & 5 & 0,210 & 28,5 & 2,38 & 2,14 & 5 & 0,210 & 2,38 & 2,14 & & & & \\
\hline 75 & 24,5 & 2 & 0,087 & 28,9 & 0,99 & 0,89 & 2 & 0,087 & 0,99 & 0,89 & & & & \\
\hline Total & & 1234 & 21,4 & & 238,30 & 203,45 & 502 & 12,8 & 145,51 & 127,04 & 732 & 8,6 & 92,79 & 76,42 \\
\hline
\end{tabular}

$V 4 c c$ e $V 4 s c=$ volumes com e sem casca até um limite superior de $4,0 \mathrm{~cm}$ de diâmetro. reman.=remanescente. desbas=desbastado.

Quadro5 - Produção baseada na distribuição de diâmetros para volume até o diâmetro mínimo igual a $15 \mathrm{~cm}$ (volume com casca e sem casca para serraria - V15cc e V15sc), considerando-se um primeiro desbaste de $40 \%$ da área basal e removendo árvores nas classes inferiores aos 88 e 75 meses, nas classes de produtividade III e I, respectivamente

Table 5 - Production based on diametric distribution for volumes up to $15 \mathrm{~cm}$ diameter minimum (volume with bark and without bark for sawmill -V15cc and V15sc), taking into consideration a first thinning of $40 \%$ basal area and removing trees in classes under 88 and 75 months, in the site classes III and I, respectively

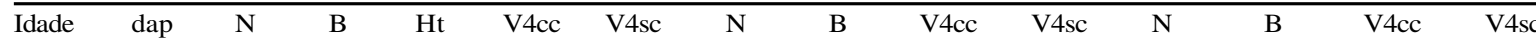

(meses) $(\mathrm{cm})$ total total total total total reman. reman. reman. reman. desbas. desbas. desbas. desbas.

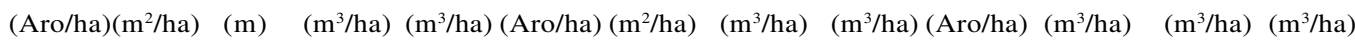

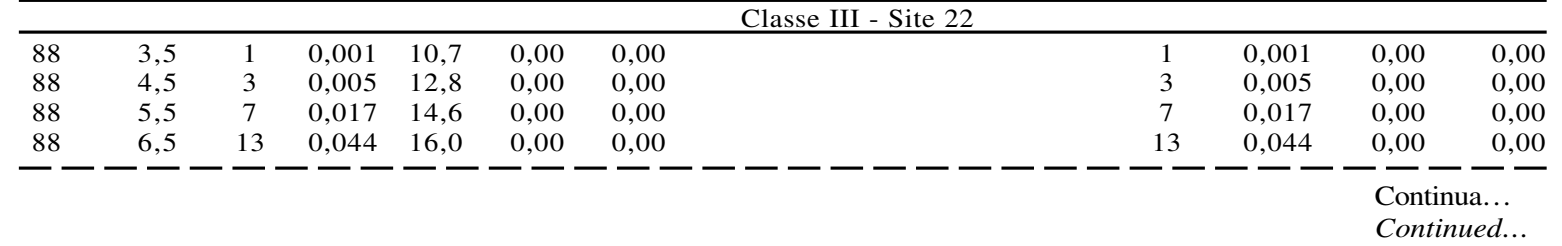

R. Árvore, Viçosa-MG, v.29, n.2, p.271-280, 2005 
Quadro 5-Cont.

Table 5 - Cont.

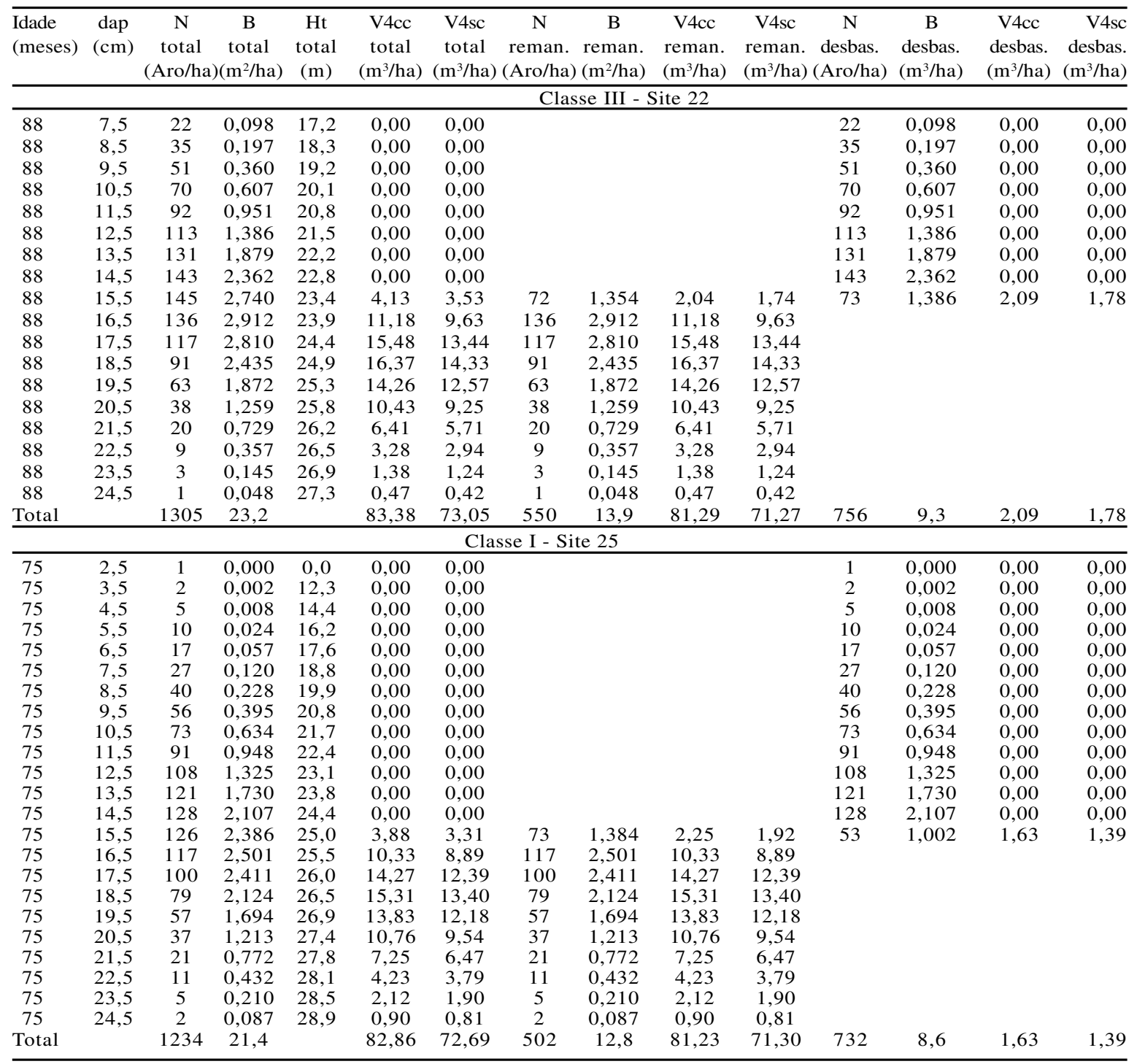

\section{CONCLUSÕES}

Os resultados permitiram concluir que:

- O modelo apresentado é biologicamente correto e consistente.

- Após o desbaste, ocorre aumento na taxa de crescimento das árvores remanescentes, resultando na estagnação mais rápida do crescimento.
- O método dos ingressos porcentuais é adequado para determinar a idade técnica do primeiro e do segundo desbaste.

- O sistema de equações proposto permite simular diferentes cenários, considerando-se, por exemplo, diversas reduções de área basal, diferentes volumes comerciais e várias idades técnicas de desbaste, resultando em estimativas consistentes. 


\section{REFERÊNCIAS BIBLIOGRÁFICAS}

BURKHART, H. E.; CAO, Q. V.; WARE, K. D. A comparison of growth and yield prediction models for loblolly pine. Blacksburg: Virginia Polytechnic Institute and State University, School of Forestry and Wildlife Resources, 1981.59 p. (Publ., FWS-2).

CAMPOS, J. C. C.; LEITE, H. G. Mensuração florestal: perguntas e respostas. Viçosa, MG: Universidade Federal de Viçosa, 2002. 407 p.

GARCIA, S. L. R. Ingresso percentual: teoria e aplicações em ciência florestal. Viçosa, MG: [1999]. 20 p. Trabalho não publicado.
GOUDRIAAN, J. Using the expolinear growth equation to analyse resource capture. In: MONTEITH, J. L.; SCOOT, R. K.; UNSWORTH, M. H. Resource capture by crops. Nottingham: University Press, 1994. p. 99-110.

NOGUEIRA, G. S. et al. Determinação da idade técnica de desbaste em plantações de eucalipto utilizando o método dos ingressos percentuais. Scientia Forestalis, n. 59, p. 51-59, 2001.

PIENAAR, L. V.; SCHIVER, B. D. Survival functions for site prepared slash pine plantations in the flat woods of Georgia Northern Florida. Southern Journal Forestry, v. 5, n. 2, p. 59-62, 1981. 